\title{
MicroRNA-135a is involved in podocyte injury in a transient receptor potential channel 1-dependent manner
}

\author{
XIANGGUI YANG, DONGMING WU, HONGFEI DU, FANG NIE, XUELI PANG and YING XU \\ Department of Laboratory Medicine, The First Affiliated Hospital of Chengdu Medical University, \\ Chengdu, Sichuan 610500, P.R. China
}

Received November 29, 2016; Accepted September 18, 2017

DOI: 10.3892/ijmm.2017.3152

\begin{abstract}
Transient receptor potential (TRP) cation channels are essential for normal cellular physiology, and their abnormal expression may lead to a number of disorders, including podocytopathy. Therefore, it is crucial to understand the mechanisms underlying the regulation of TRP channels. In the present study, microRNA (miR)-135a was found to be upregulated in patients with focal segmental glomerulosclerosis and mice treated with adriamycin (ADR). In cultured podocytes, transforming growth factor (TGF) $\beta$ and ADR were found to promote miR-135a expression. Conversely, TRP channel 1 (TRPC1) protein levels were markedly downregulated in podocytes from mice treated with ADR, as well as in cultured podocytes treated with ADR and TGF- $\beta$. Ectopic expression of miR-135a led to severe podocyte injury and disarray of the podocyte cytoskeleton, whereas podocyte-specific expression of TRPC1 was able to reverse the pathological effects of miR-135a in cultured podocytes. Moreover, using Luciferase reporter assays and western blot analysis, TRPC1 was identified as a target gene of miR-135a. To the best of our knowledge, this is the first study to demonstrate the role of TRPC1 in the development of podocyte injury and disorders of the podocyte cytoskeleton, which may contribute to the development of novel therapeutics for podocyte injury-associated kidney diseases.
\end{abstract}

\section{Introduction}

Transient receptor potential (TRP) proteins are non- selective cation channels performing numerous roles as versatile cellular sensors and effectors (1). The TRP superfamily consists of 33 channels, which are divided into seven families: TRPC, TRPM, TRPV, TRPA, TRPP, TRPML and TRPN. Apart from the TRPN channels, all TRP families are expressed in humans

Correspondence to: $\mathrm{Ms}$. Ying $\mathrm{Xu}$, Department of Laboratory Medicine, The First Affiliated Hospital of Chengdu Medical College, 278 Baoguang Road, Xindu, Chengdu, Sichuan 610500, P.R. China

E-mail: yingxu825@126.com

Key words: microRNA-135a, podocyte, injury, transient receptor potential channel 1 and are associated with diverse conditions, such as neurological, cardiological, pulmonary, renal, dermatological and urological diseases (1). TRPC1, the founding member of the TRPC family, was the first cloned mammalian TRP protein, but its molecular function as a channel in the development of kidney disease, particularly podocytopathy, remains obscure.

Podocytopathy, including podocyte injury and loss, is a major pathological mechanism underlying glomerular sclerosis (2). Although numerous signaling pathways have been implicated in podocyte damage $(3,4)$, it has been demonstrated that microRNAs are necessary for podocyte homeostasis, with podocyte-selective deletion of dicer, an enzyme that generates microRNAs (5), promoting podocyte injury $(6,7)$. More recently, several microRNAs, including miR-193a (8), miR-29a (9), the miR-30 family $(10,11)$ and the miR-135 family (12), have been implicated in podocyte injury.

The miR-135 family is evolutionarily highly conserved and comprises two members, miR-135a and miR-135b. It has been reported that miR-135a functions as a tumor suppressor gene in gastric (13), prostate (14) and renal cancer (15), and malignant glioma cells (16). However, other studies recently demonstrated that miR-135a acts as a tumor-promoting gene involved in the development of several cancers, including the pathogenesis of colorectal cancer (17), the promotion of paclitaxel resistance in non-small-cell lung cancer (18), and the facilitation of growth and invasion in colorectal cancer (19). In addition, it was demonstrated that ectopic expression of miR-135 family members may induce actin fiber disruption (12), suggesting that miR-135a is involved in podocyte actin fiber and cytoskeletal stability via an undetermined mechanism. Despite promising findings, the precise function of the miR-135a remains largely unknown, particularly in podocyte injury-associated renal diseases.

The aim of the present study was to determine the role and mechanisms of action of miR-135a and TRPC1 in podocyte injury, and to elucidate the mechanisms underlying this type of injury. miR-135a was found to be overexpressed in patients with focal segmental glomerular sclerosis (FSGS) and in models of podocyte injury; in addition, the ectopic expression of miR-135a promoted podocyte injury by downregulating TRPC1 expression. Our findings originally demonstrated that miR-135a and TRPC1 play an important role in podocyte injury, and may provide novel insight into the understanding of the molecular mechanisms underlying podocyte injury, which 
may be crucial for the development of novel therapeutic agents for the treatment of podocytopathy.

\section{Materials and methods}

Human samples. A total of 3 patients with FSGS were diagnosed by renal biopsy at the Department of Nephrology of the First Affiliated Hospital of Chongqing Medical University (Chongqing, China). The control group comprised patients with kidney rupture following incidents of violence or traffic accidents. The glomeruli were collected from the renal tissues using the sieving technique, as previously described (20), placed in TRIzol reagent (Ambion, Austin, TX, USA) and stored at $-70^{\circ} \mathrm{C}$ for RNA extraction and molecular analysis. Human subject research approval was granted by the Ethics Committee of the First Affiliated Hospital of Chongqing Medical University. All the participants enrolled in this study provided written informed consent.

Animal experiments. The use of animals in the present study was approved by the Ethics Committee of the First Affiliated Hospital of Chongqing Medical University. BALB/c mice (6-7 weeks old, weighing 24-28 g, $n=60$ ) were used to create the adriamycin (ADR)-induced podocyte injury model. The models were generated by administration of an intravenous injection of ADR (10.5 mg/kg; Sigma-Aldrich; Merck KGaA, St. Louis, MO, USA) as previously described $(21,22)$. For the control group, the mice received an equivalent volume of normal saline. The mice were sacrificed by cervical vertebral dislocation on days 4, 7 , 11,15 and 20 following the administration of ADR or normal saline. In total, there were 10 groups (5 ADR-treated groups and 5 normal saline-treated groups) with 6 mice in each; one ADR-treated group and one normal saline-treated group were sacrificed at the indicated time points (4, 7, 11, 15 and 20 days). The glomeruli were isolated from the kidneys using the sieving technique, as previously described (20), and were snap-frozen in liquid nitrogen for RNA and protein extraction.

Cell culture and treatment. The mouse podocyte cell line 5 (MPC5) was used, and the cells were cultured as previously described (23). In brief, the cells were cultured at $33^{\circ} \mathrm{C}(10 \mathrm{U} / \mathrm{ml}$ of interferon- $\gamma)$ with $5 \% \mathrm{CO}_{2}$ and were differentiated at $37^{\circ} \mathrm{C}$ (free interferon- $\gamma$ ) with $5 \% \mathrm{CO}_{2}$ in RPMI Dutch-modified medium (Invitrogen; Thermo Fisher Scientific, Carlsbad, CA, USA) supplemented with $10 \%$ v/v fetal calf serum (Gibco-BRL; Thermo Fisher Scientific, Carlsbad, CA, USA). 293T cells were cultured in Dulbecco's modified Eagle's medium (Invitrogen; Thermo Fisher Scientific) supplemented with $10 \% \mathrm{v} / \mathrm{v}$ fetal bovine serum at $37^{\circ} \mathrm{C}$ with $5 \% \mathrm{CO}_{2}$. Depending on the experimental setup, the MPC5 cells were treated with different doses of ADR and transforming growth factor (TGF)- $\beta$. For transient transfection of miRNA mimics and plasmid DNA, Lipofectamine 3000 reagent (Invitrogen; Thermo Fisher Scientific, Grand Island, NY, USA) was used, according to the manufacturer's instructions.

Bioinformatics analysis. Predicted miR-135a targets were obtained from miRwalk (www.umm.uni-heidelberg.de/apps/ zmf/mirwalk), miRanda (www.microrna.org/microrna/home. do) and TargetScan (www.targetscan.org/).
Plasmid construction. Genomic DNA fragments containing the $3^{\prime}$ untranslated region (UTR) of TRPC1 were prepared by the amplification of genomic DNA, cloned onto the pcDNA3.1-Luc reporter vector and verified by sequencing. For the wild-type construct, the seed sequence was wild-type. For the mutant construct, the seed sequence was mutated. The TRPC1 coding sequence (CDS) was obtained by polymerase chain reaction (PCR) and cloned into the pcDNA3.1(+) plasmid. The sequences of the primers used are listed in Table I.

Luciferase assays. miR-135a mimics and miRNA mimics negative control were purchased from RiboBio Co., Ltd. (Guangzhou, China). Luciferase assays were conducted as previously described (24). In brief, the $293 \mathrm{~T}$ cells were cultured in 24-well plates and transfected with $500 \mathrm{ng}$ of the reporter vector, $50 \mathrm{ng}$ of $\mathrm{pRL}-\mathrm{CMV}$ and $50 \mathrm{nM}$ of miR-135a mimics or the miRNA mimics negative control (RiboBio Co., Ltd.) using Lipofectamine 3000 reagent (Invitrogen; Thermo Fisher Scientific). According to the dual-Luciferase assay system manufacturer's instructions (Promega, Madison, WI, USA), the Firefly and Renilla Luciferase activities were measured at 36-48 $\mathrm{h}$ after transfection.

RNA extraction and reverse transcription-quantitative polymerase chain reaction ( $R T-q P C R)$. Total RNA was extracted from the cultured cells and renal tissue with TRIzol reagent (Ambion), according to the manufacturer's instructions. cDNA was synthesized from $5 \mathrm{ng}$ of total RNA with the RevertAid First Strand cDNA synthesis kit (Fermentas, Burlington, ON, Canada). RT-qPCR for the detection of miR-135a was performed using miR-135a-specific PCR primers (RiboBio Co., Ltd.) with the RevertAid First Strand cDNA Synthesis kit (Fermentas) and SYBR Premix Ex Taq ${ }^{\mathrm{TM}}$ II (Takara, Dalian, China) according to the manufacturers' instructions, with U6 as the internal control. The sequences of the primers used in RT-qPCR are presented in Table II. $18 \mathrm{~S}$ was used as the internal control.

Protein extraction and western blot analysis. Total protein was extracted from the MPC5 cells and isolated glomeruli using radioimmunoprecipitation assay lysis buffer (Beyotime, Jiangsu, China) according to the manufacturer's instructions. Western blot analysis was performed with a standard method, as previously described (25). The antibodies used were as follows: Rabbit anti-desmin (1:1,000; sc-14026), rabbit anti-snail (1:1,000; sc-28199), rabbit anti-TRPC1 (1:1,000; sc-28199), rabbit anti-Wilms tumor 1 (WT1; 1:500; sc-192), rabbit anti-Ecadherin $(1: 1,000 ;$ sc-7870), mouse anti- $\beta$-tubulin $(1: 3,000$; sc-80011), goat anti-rabbit IgG-HRP (1:3,000; sc-2004), goat anti-mouse IgG-HRP (1:3,000; sc-2005) (all from Santa Cruz Biotechnology, Inc., Santa Cruz, CA, USA), and rabbit anti-nephrin (1:2,000; ab136894; Abcam, Cambridge, MA, USA). Images were captured with a SPOT CCD camera (Diagnostic, Sterling Heights, MI, USA). For the quantitative analysis of the western blots, the protein band intensities were quantified using ImageJ software and $\beta$-tubulin was used as the internal control.

F-actin cytoskeleton staining and immunofluorescence staining. Rhodamine-labeled phalloidin (diluted in phosphate-buffered saline (PBS) containing 5\% bovine serum 
Table I. Sequences of the PCR primers for construction.

\begin{tabular}{lc}
\hline Name & \multicolumn{1}{c}{ Primer sequences } \\
\hline $\begin{array}{l}\text { TRPC1 3'UTR } \\
\text { Sense }\end{array}$ & 5'-TGTATTTGCATACTTGCAA-3' \\
Antisense & 5'-GAGGGAACACTTAAACCTG-3' \\
TRPC1 3'UTR & \\
(mutant) & \\
Sense & 5'-TGTATTTGCATACTTGCAA-3' \\
& 5'-CAATTACACATACGGCTTTCTT-3' \\
Antisense & 5'-AAGAAAGCCGTATGTGTAATTG-3' \\
& 5'-GAGGGAACACTTAAACCTG-3' \\
TRPC1 CDS & \\
Sense & 5'-ATGGGGGCCCCGCCTCCGTCT-3' \\
Antisense & 5'-TTAATTTCTTGGATAAAACATAG-3' \\
\hline
\end{tabular}

PCR, polymerase chain reaction; TRPC1, transient receptor potential channel 1; UTR, untranslated region; CDS, coding sequence.

Table II. Sequences of RT-PCR primers.

\begin{tabular}{lcc}
\hline Name & Primer sequences & $\begin{array}{c}\text { Product } \\
\text { size (bp) }\end{array}$ \\
\hline TRPC1 & & \\
Sense & 5'-GGATTATTGGGATGATTTG-3' & 143 \\
Antisense & 5'-GTGAGCCACCACTTTGAG-3' & \\
18S & & \\
Sense & 5'-GTAACCCGTTGAACCCCATT-3' & 151 \\
Antisense & 5'-CCATCCAATCGGTAGTAGCG-3' & \\
& &
\end{tabular}

RT-PCR, reverse trancription-polymerase chain reaction; TRPC1, transient receptor potential channel 1 .

albumin, 1:1,000; Sigma-Aldrich; Merck KGaA) was used to strain F-actin according to the manufacturer's instructions. Images were captured with a SPOT CCD camera (Diagnostic).

Flow cytometric analysis of apoptosis via Annexin V staining. After treatment, the cultured podocytes were collected and washed with ice-cold PBS twice, resuspended in $200 \mu \mathrm{l}$ binding buffer, and then incubated with FITC-conjugated Annexin V (final concentration, $0.5 \mu \mathrm{g} / \mathrm{ml}$ ) for $15 \mathrm{~min}$ at room temperature. Subsequently, the cells were again washed with ice-cold PBS, centrifuged, and resuspended in $500 \mu \mathrm{l}$ binding buffer. Finally, $50 \mu \mathrm{g} / \mathrm{ml}$ propidium iodide was used to stain the cells at room temperature for $5 \mathrm{~min}$, followed by flow cytometric analysis using a FACScan flow cytometer and CellQuest software (BD Biosciences, Franklin Lakes, NJ, USA).

Statistical analysis. All the data are presented as means \pm standard deviation. The two-tailed Student's t-test was employed to indicate whether two sets of data (groups) differed significantly using GraphPad Prism 5 software (GraphPad Software,
Inc., La Jolla, CA, USA). P-values $<0.05$ and $<0.01$ were considered statistically significant and highly statistically significant, respectively. All the experiments were performed at least 3 times.

\section{Results}

Enhanced miR-135a expression in podocyte injury. To investigate whether miR-135a is involved in the pathogenesis of podocyte injury, glomeruli were first isolated from 3 patients with FSGS, whose podocytes were severely damaged, and from 3 patients who had suffered kidney rupture due to incidents of violence or traffic accidents. The ruptured kidney samples were used as controls. miR-135a expression was examined by RT-qPCR in the glomeruli from these patients and, as indicated in Fig. 1A, miR-135a was highly expressed in the glomeruli of FSGS patients compared with those of controls. Furthermore, a mouse model was established by intravenous injection of ADR $(10.5 \mathrm{mg} / \mathrm{kg})$, which is a widely recognized model of podocyte injury $(21,22)$, and the expression of miR-135a was then detected in the isolated glomeruli. The expression level increased significantly as early as day 4 following the injection of ADR. On day 15 following treatment with ADR, the miR-135a expression level improved by $\sim 3$-fold. Additionally, the expression level of miR-135a was measured in in vitro models of ADR-induced and TGF- $\beta$-induced podocyte injury. As illustrated Fig. 1C-F, treatment with ADR increased the expression of miR-135a in cultured MPC5 cells in a dose-dependent manner [24 h after ADR treatment (Fig. 1C)], as well as in a time-dependent manner [ $5 \mu \mathrm{g} / \mathrm{ml}$ ADR treatment (Fig. 1D)]. Similarly, TGF- $\beta$ enhanced the expression level of miR-135a in a dose-dependent manner [24 $\mathrm{h}$ after TGF- $\beta$ treatment (Fig. 1E)], as well as in a time-dependent manner [ $5 \mathrm{ng} / \mathrm{ml} \mathrm{TGF-} \beta$ treatment (Fig. $1 \mathrm{~F}$ )] in cultured MPC5 cells. Thus, these data clearly demonstrated that miR-135a was upregulated in podocyte injury models and glomeruli isolated from patients with FSGS.

miR-135a promotes podocyte injury and apoptosis. As shown in Fig. 1, our results demonstrated that miR-135a expression was upregulated in podocyte injury. We then determined whether the increased expression of miR-135a was functionally significant in podocyte damage. miR-135a mimics and miRNA mimics negative control were transfected into the cultured MPC5 cells. As indicated in Fig. 2A and B, the protein expression levels of desmin and Snail, two markers associated with podocyte injury, were significantly increased in the cells transfected with miR-135a mimics compared with the cells transfected with miRNA mimics negative control. However, the expression of functional markers, such as nephrin, WT1 and E-cadherin, was inhibited by miR-135a mimics (Fig. 2A and B). To further support the hypothesis of the damaging effect of miR-135a on podocytes, at $48 \mathrm{~h}$ following miR-135a mimics transfection into MPC5 cells, the podocyte cytoskeleton was examined by means of fluorescein-conjugated phalloidin staining, and it was observed that the stress fibres in podocytes were severely reduced in number and disordered (Fig. 2C). The podocytes of cells transfected with miR-135a mimics also exhibited significantly increased levels of apoptosis compared with miRNA negative-transfected control cells (Fig. 2D). Furthermore, the 


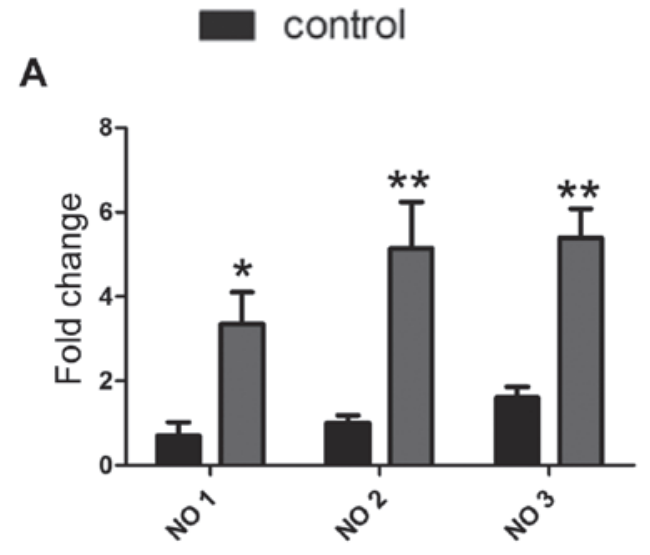

C

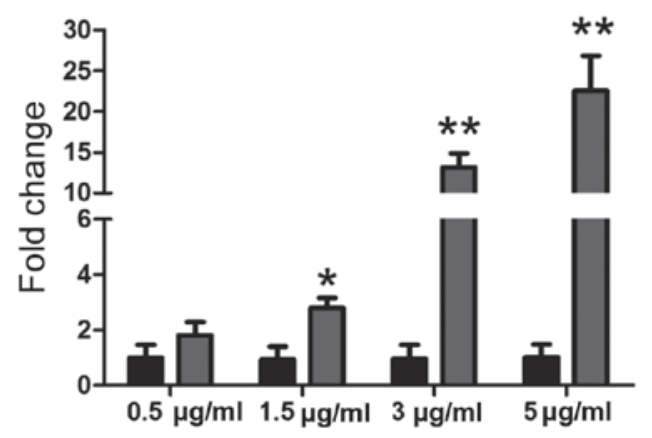

E

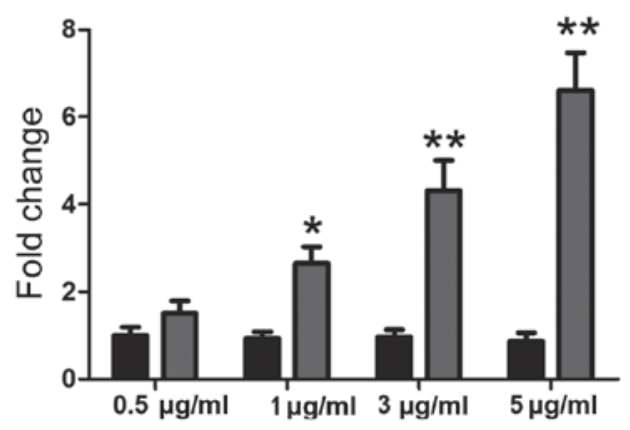

\section{ADR/TGF- $\beta$-treated or FSGS}

B

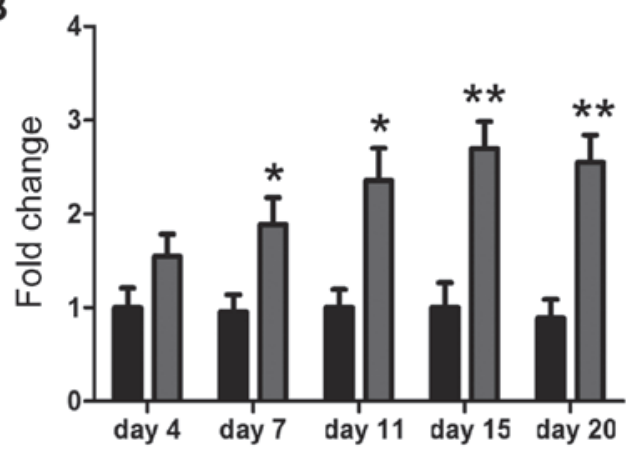

D

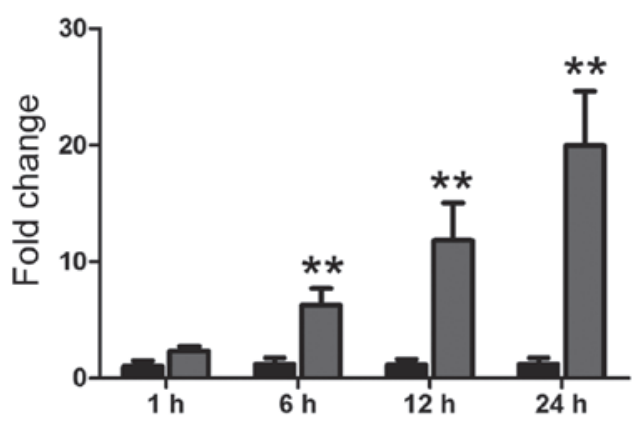

$\mathbf{F}$

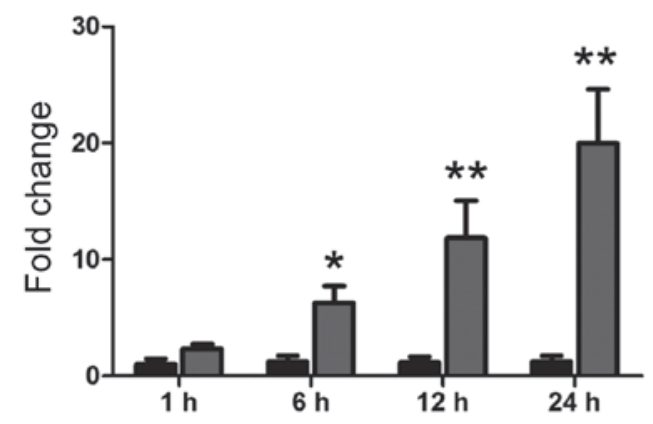

Figure 1. miR-135a is upregulated in podocyte injury. (A) Quantification by reverse transcription-quantitative polymerase chain reaction (RT-qPCR) of miR-135a in the isolated glomeruli from 3 patients with focal segmental glomerulosclerosis (FSGS) and 3 patients with kidney rupture (control). (B) RT-qPCR analysis of miR-135a expression in the glomeruli isolated from BALB/c mice treated with adriamycin (ADR; $10.5 \mathrm{mg} / \mathrm{kg}$ ) at different time points after the injection [n=3, means \pm standard deviation (SD)]. (C and D) The miR-135a expression level was measured in the cultured mouse podocyte cell line 5 (MPC5) treated with ADR (C) at different doses and (D) for different periods of time. (E and F) Quantification of miR-135a in MPC5 cells treated with transforming growth factor (TGF) $\beta(\mathrm{E})$ at different doses and $(\mathrm{F})$ for different periods of time. The data are expressed as mean $+\mathrm{SD}$ of three independent experiments. ${ }^{*} \mathrm{P}<0.05$ and ${ }^{* *} \mathrm{P}<0.01$ indicate statistically significant and highly statistically significant differences, respectively, compared with the controls. NO1, patient number 1 ; NO2, patient number 2; NO3, patient number 3.

ectopic expression of miR-135a improved the protein expression level of activated caspase-3 (Fig. 2A and B). These date clearly indicated that miR-135a enhances podocyte injury and promotes podocyte apoptosis.

The expression level of TRPC1 was downregulated in podocyte injury. In order to further elucidate the molecular mechanisms underlying miR-135a-induced podocyte injury, the targets of miR-135a were predicted by online miRNA target prediction programmes, such as TargetScan (www.targetscan.org) PicTar (http://pictar.mdc-berlin.de) and miRanda (www.microrna.org), suggesting that miR-135a possessed a highly conserved binding site in the 3'UTR of
TRPC1 (Fig. 3A). Subsequently, the expression level of TRPC1 was investigated. As shown in Fig. 3B and C, TRPC1 expression was severely inhibited in the glomeruli isolated from ADR-induced podocyte injury mouse models; on day 20 following ADR injection, the expression level of TRPC1 had decreased by $75 \%$ compared with the control group. In cultured MPC5 cells, the expression level of TRPC1 was reduced by ADR and TGF- $\beta$ (Fig. 3D and E).

miR-135a directly targets TRPC1 3'UTR and inhibits TRPC1 expression. In order to further validate the results of bioinformatics analysis, mouse TRPC1 3'UTR was cloned into the pcDNA3.1-Luciferase reporter vector, generating a mutant 
A

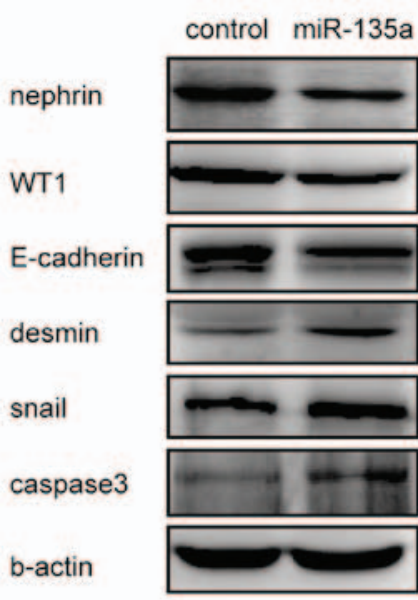

C

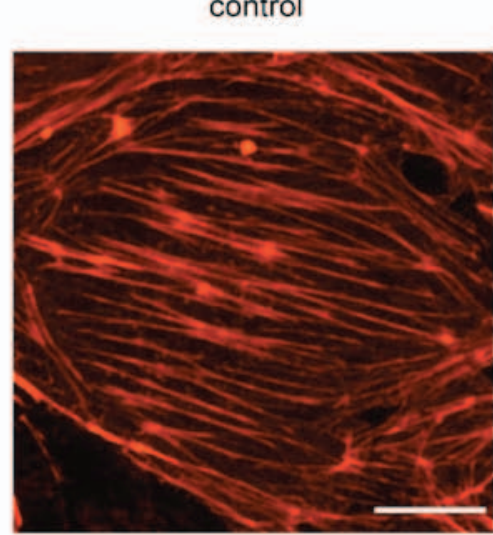

D

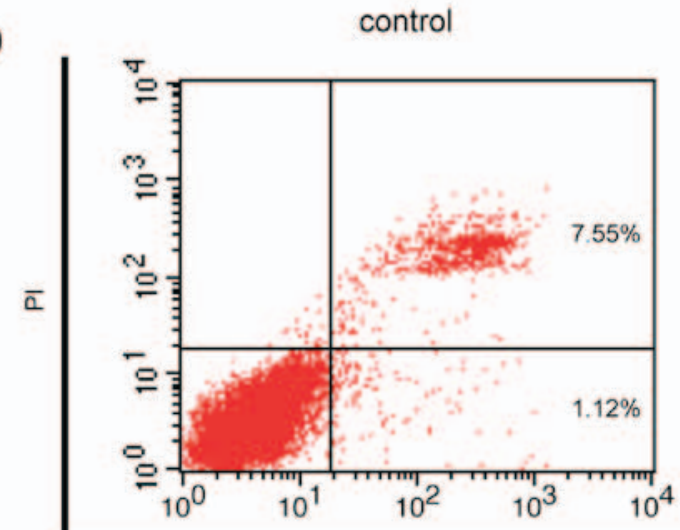

B

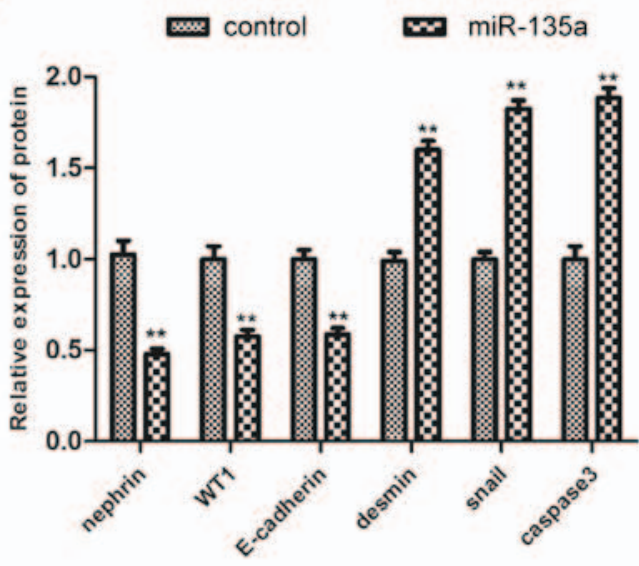

miR-135a

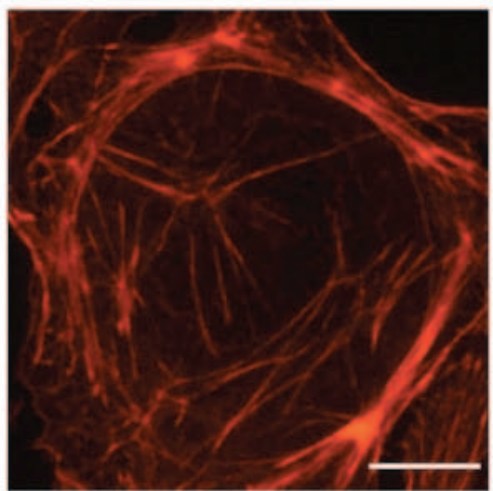

miR-135a

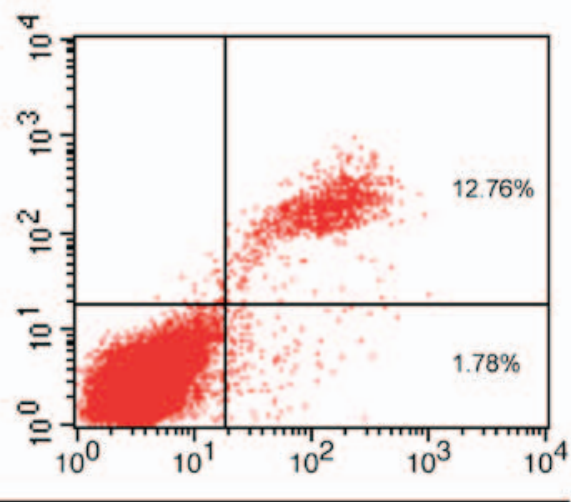

Annexin V-FITC

Figure 2. Ectopic expression of miR-135a led to severe podocyte injury. (A) The changes in the expression levels of nephrin, Wilms tumor 1 (WT1), E-cadherin, desmin, Snail and activated caspase-3 were determined by western blot analysis in the cultured MPC5 cells treated with miR-135a mimics. (B) Quantitative analysis of the results of western blot analysis. (C) Effects of miR-135a on cytoskeletal stabilization in podocytes. Scale bar, $10 \mu \mathrm{m}$. (D) MPC5 cell apoptosis was detected by flow cytometry following transfection of miR-135a mimics. ${ }^{*} \mathrm{P}<0.05$ and ${ }^{* * *} \mathrm{P}<0.01$ indicate statistically significant and highly statistically significant differences, respectively, compared with the control group.

reporter vector in which the putative miR-135a binding site AAAGCCAU in the TRPC1 3'UTR was mutated into ATA CGGCTU (Figs. 3A and 4A and B). Luciferase reporter assays were conducted in HEK293T cells to determine whether miR-135a significantly repressed TRPC1 transcript levels. The results revealed that Luciferase expression was significantly suppressed in the group transfected with the miR-135a mimics compared with miRNA mimics negative control group, while mutations of the miR-135a-binding sites reversed the suppression of Luciferase expression (Fig. 4C). Moreover, the effect of miR-135a on TRPC1 expression was also examined, and the results indicated that miR-135a inhibited the expression of TRPC1 at the protein as well as at the mRNA level (Fig. 4D-F). Taken together, these results demonstrated that miR-135a directly binds to the 3'UTR of TRPC1 and inhibits TRPC1 expression. 
A

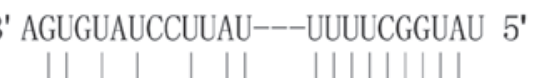

mouse miR-135a

5' AUGGGCAUAAACAAUUACACAAAAGCCAUUCUUUAAA 3' mouse TRPC1-3'UTR

- || ||| ||||| |||||||||||||||||

5' AUUGAAAUAAAGAAUUAUGUAAAAGCCAUUCUUUAAA 3' human TRPC1-3'UTR

3' AGUGUAUCCUUAU---UUUUCGGUAU 5'

human miR-135a

B

control day4 day7 day11 day14 day20

TRPC1

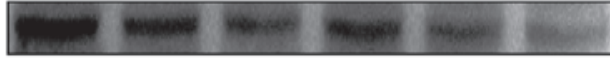

$\beta$-tubulin

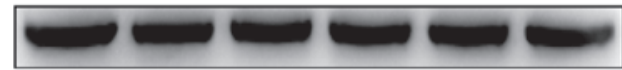

D

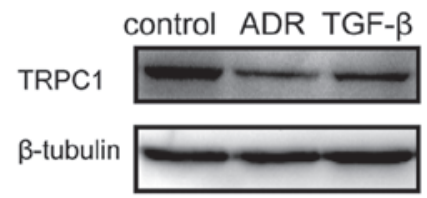

C

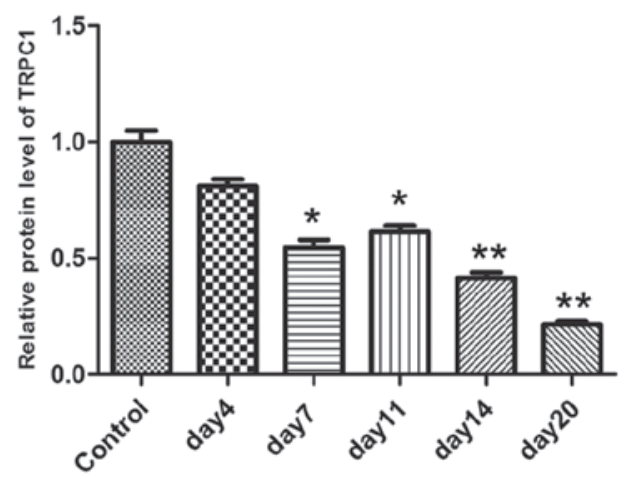

E

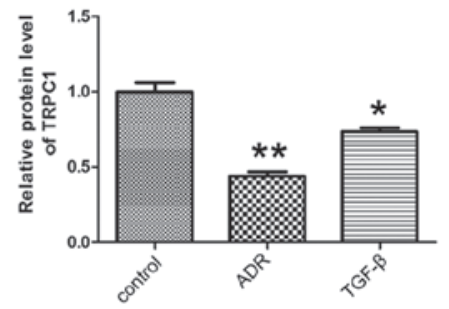

Figure 3. Transient receptor potential channel 1 (TRPC1) expression was downregulated in podocyte injury. (A) Predicted miR-135a target TRPC1 3' untranslated region (UTR) in human and mouse species. (B) The protein level of TRPC1 was downregulated in the glomeruli isolated from BALB/c mice treated with adriamycin (ADR) in a time-dependent manner. (C) Quantitative analysis of the results of (B). (D) TRPC1 expression was inhibited in cultured MPC5 cells treated with ADR $\left(5 \mu \mathrm{g} / \mathrm{ml}, 24 \mathrm{~h}\right.$ ) and transforming growth factor (TGF)- $\beta(5 \mathrm{ng} / \mathrm{ml}, 24 \mathrm{~h})$. (E) Quantitative analysis of the results of (D). ${ }^{*} \mathrm{P}<0.05$ and ${ }^{* *} \mathrm{P}<0.01$ indicate statistically significant and highly statistically significant differences, respectively, compared with the control group.

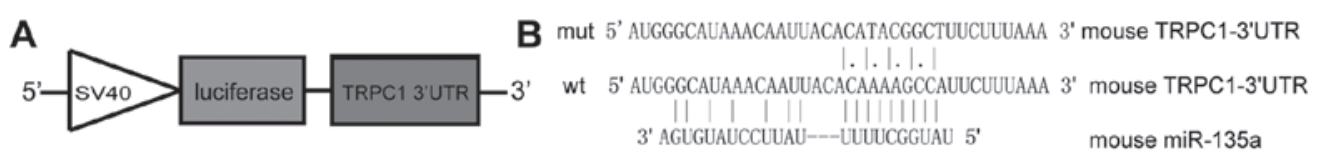

C

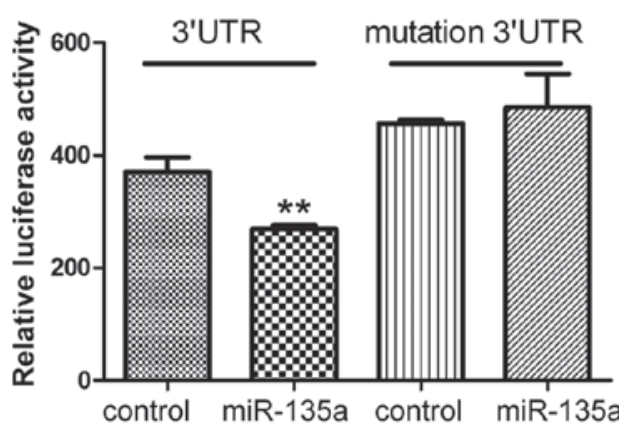

$\mathbf{E}$

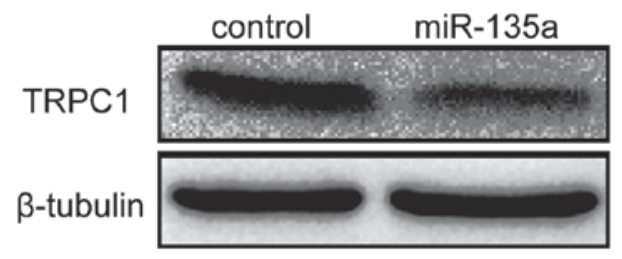

D

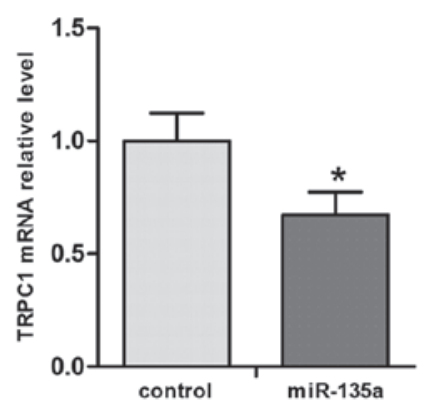

$\mathbf{F}$

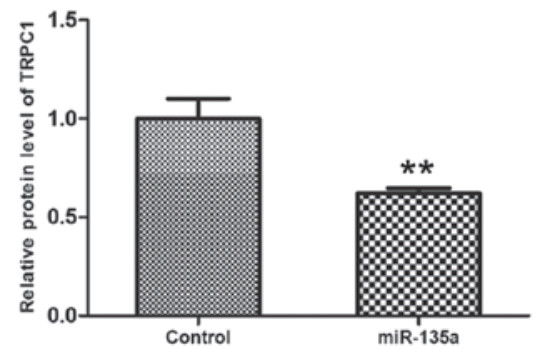

Figure 4. miR-135a directly targets transient receptor potential channel 1 (TRPC1) 3' untranslated region (UTR). (A) Schematic diagram of TRPC1 3'UTR reporter construct. (B) Sequence alignment between miR-135a and mouse TRPC1 3'UTR, including wild-type (wt) and mutant (mut). (C) For dual Luciferase reporter assays, 293T cells were transfected with pCDNA3.1-Luciferase TRPC1-3'UTR reporter and miR-135a mimics. (D) Reverse transcription-quantitative polymerase chain reaction analysis was used to determine the effect of miR-135a on TRPC1 mRNA expression. (E) The effect of miR-135a on TRPC1 protein expression was determined by western blot analysis. (F) Quantitative analysis of the results of (E). ${ }^{*} \mathrm{P}<0.05$ and ${ }^{* *} \mathrm{P}<0.01$ indicate statistically significant and highly statistically significant differences, respectively, compared with the controls. 
A

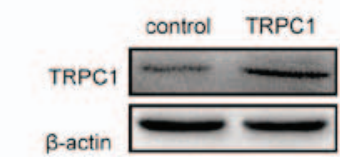

B

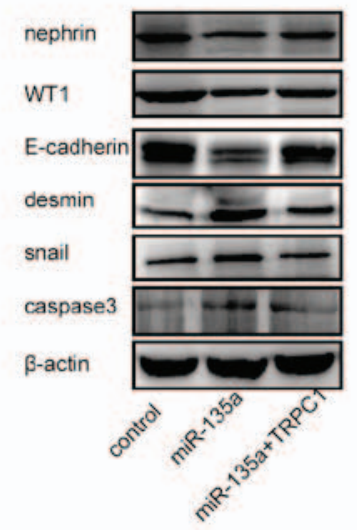

D

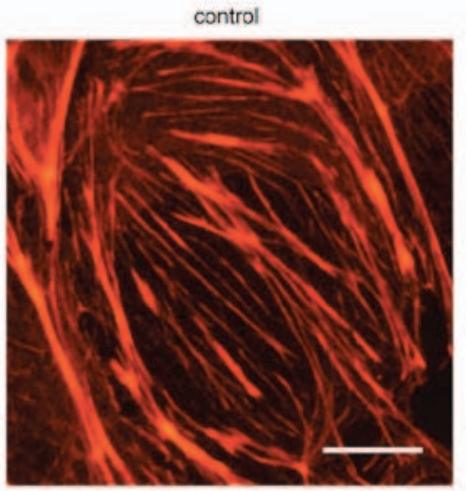

E

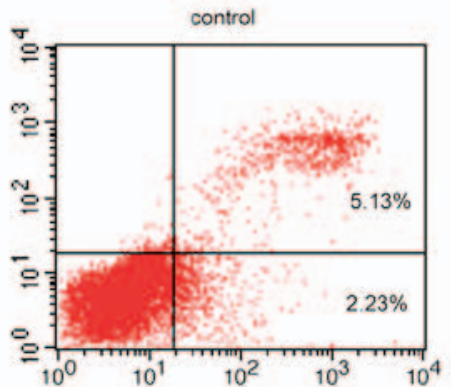

C

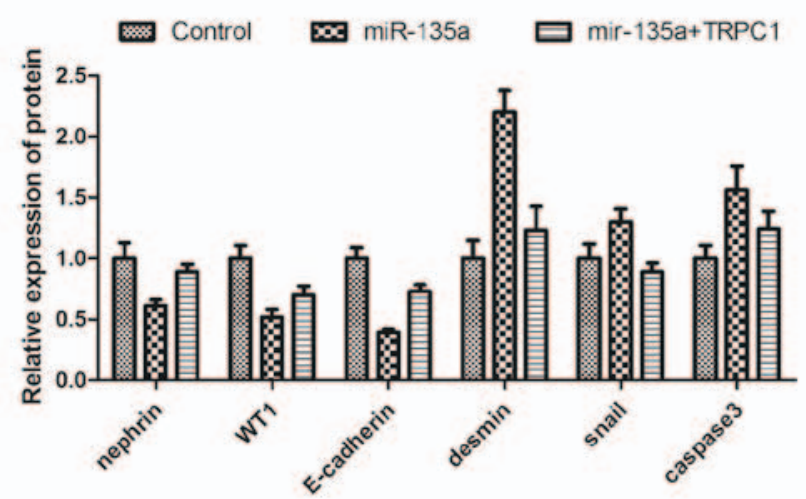

$m i R-135 a$
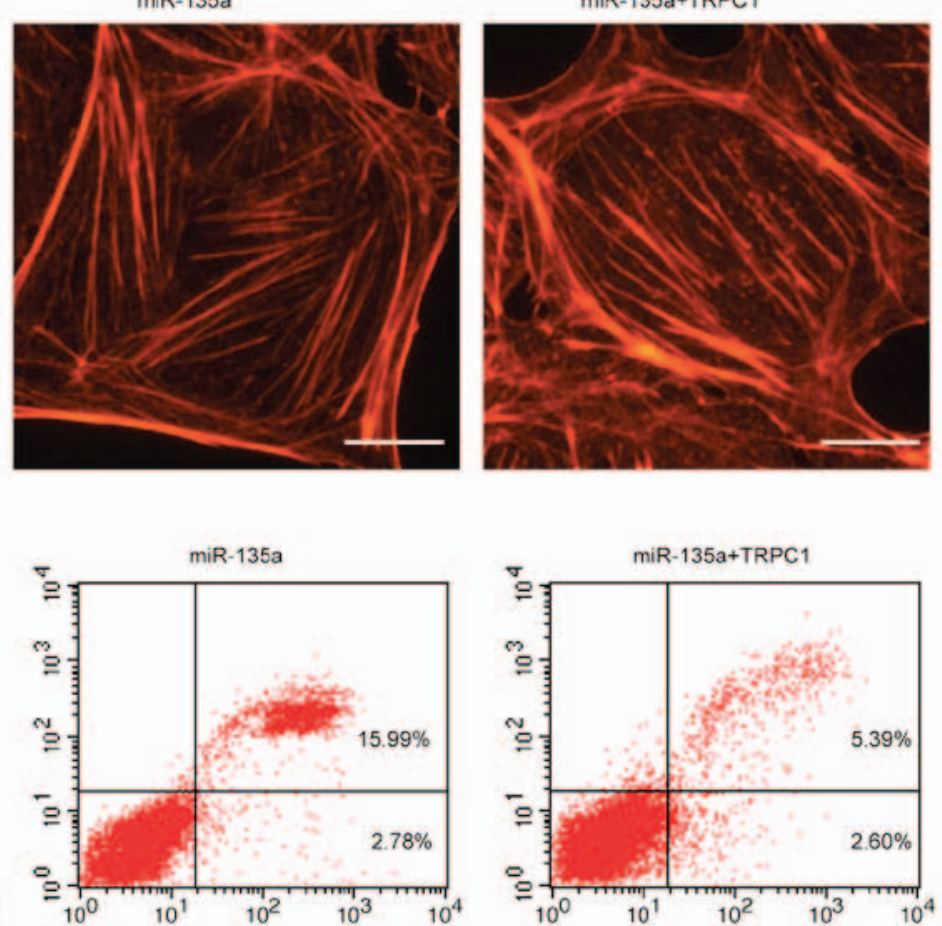

Figure 5. Ectopic expression of transient receptor potential channel 1 (TRPC1) protects against podocyte damage from miR-135a. (A) Western blot analysis confirmed that the TRPC1 overexpression construct was available. (B) Western blot analysis was applied to determine the effects on the expression levels of nephrin, Wilms tumor 1 (WT1), E-cadherin, desmin, Snail and activated caspase-3 in MPC5 cells transfected with miR-135a and/or TRPC1 overexpression construct. (C) Quantitative analysis of the results of (B). (D) Stress fibre reduction in number and disarray, which was induced by miR-135a, was reversed by overexpression of TRPC1. Scale bar, $10 \mu \mathrm{m}$. (E) Ectopic expression of TRPC1 alleviated miR-135a-induced podocyte apoptosis.

Overexpression of TRPC1 abrogates miR-135a-induced podocyte injury. To investigate whether the miR-135a-induced podocyte injury was TRPC1-dependent, mouse TRPC1 CDS was cloned into the pcDNA3.1(+) vector (TRPC1 overexpression vector). Western blot analyses confirmed that TRPC1 was significantly increased by ectopic expression compare with control (Fig. 5A). The expression of podocyte injury markers and functional markers was next determined, and the results suggested that TRPC1 reversed the increased expression of desmin/Snail/activated caspase-3, and inhibited the miR-135a-induced expression of nephrin/WT1/E-cadherin (Fig. 5B and C). Furthermore, the effect of miR-135a on the podocyte cytoskeleton was investigated, and the results indicated that the miR-135a-induced stress fibre decrease in number and disarray was reversed by overexpression of TRPC1. Additionally, in order to further investigate the significance of TRPC1 in podocyte injury, miR-135a mimics and the TRPC1 overexpression vector were co-transfected into cultured MPC5 cells, and the apoptosis ratio was measured with flow cytometry; the results indicated that miR-135a induced severe podocyte apoptosis, which was reversed by ectopic expression of TRPC1 (Fig. 5E). Therefore, miR-135a induced podocyte injury in a TRPC1-dependent manner. 


\section{Discussion}

The findings of the present study, consistently with those of previous studies (12), indicated that miR-135a is an important signature microRNA in podocyte injury, and its expression was significantly upregulated in glomeruli isolated from FSGS patients and podocyte injury mouse models, as well as in cultured cells treated with ADR and TGF- $\beta$. This finding was associated with the development of kidney diseases, particularly glomerulopathies; thus, miR-135a may be a potential diagnostic biomarker for glomerular and podocyte injury. More importantly, our study indicated that ectopic expression of miR-135a promotes podocyte injury, in terms of downregulating functional markers, upregulating injury marker, reducing the number of stress fibres and promoting apoptosis, which suggested that targeting miR-135a may be a viable approach to preventing the progression of podocyte injury.

Moreover, TRPC1 was identified as a target of miR-135a during podocyte injury, and overexpression of TRPC1 was able to reverse the damaging effects of miR-135a on podocytes. These results are significant, as the expression of TRPC1 is inhibited in several types of kidney disease and animal models of kidney disease, such as nodular FSGS (26), as well as in animal models of diabetes (26-28). Furthermore, it was recently demonstrated that TRPC1 genetic polymorphisms are involved in diabetic nephropathy in type 2 diabetes in Chinese patients, and that TRPC1 is a strong positional and biological candidate for diabetic nephropathy $(26,28)$. Therefore, we predicted that miR-135a-induced downregulation of TRPC1 may be a major mechanism involved in podocyte injury. Of note, it was demonstrated that overexpression of TRPC1 protects against podocyte injury from miR-135a. Thus, TRPC1 may ameliorate progression of podocyte damage.

Wu et al previously suggested that miR-135a significantly downregulated Luciferase activity in wild-type as well as mutant TRPC1 3'UTR, which suggested that miR-135a may not directly target TRPC1 3'UTR to inhibit TRPC1 expression (16). Thus, it is worth considering whether there is any association between the location of TRPC1 genetic variants and the binding site of miR-135a. Our results confirmed the ability of miR-135a to reduce TRPC1 expression by directly binding to the TRPC1 3'UTR. A novel mutant of TRPC1 3'UTR was generated, which included the putative miR-135a-binding site, and demonstrated that miR-135a significantly inhibited the Luciferase activity of TRPC1 3'UTR, without affecting the mutant TRPC1 3'UTR. Thus, our data indicated that TRPC1 is a direct target of miR-135a.

However, the precise mechanism underlying miR-135a-induced podocyte injury may also be associated with other putative target genes of miR-135a. A recent study demonstrated that miR-135a may activate the Wnt signaling pathway through inhibiting GSK- $\beta$ (12). In addition, studies on miR-135a suggested that it modulates multiple target genes, including MTSS1 (29), HOXA10 (30), SMAD5 (31) and JAK2 (32). Our data indicated that TGF- $\beta 1$ induced the miR-135a-mediated reduction in TRPC1 expression, suggesting that miR-135a may function downstream of TGF- $\beta 1$ signaling, promoting the progression of podocyte injury in kidney diseases. Additionally, TRP channels are expressed along the nephron and play an important role in kidney function (33). Using online miRNA target prediction programmes, such as TargetScan, PicTar and miRanda, we predicted that the TRP superfamily members TRPC6, TRPM4 and TRPM7 may be potential targets of miR-135a. TRPC6, a glomerular slit diaphragm protein, is necessary for normal renal function (34). TRPM4 and TRPM7 are $\mathrm{Mg}^{2+}$ transport channels and are ubiquitously expressed in the kidney (33). Taken together, these findings indicate that miR-135a may regulate TRPs members collectively to affect podocyte physiological processes, and abnormal regulation of miR-135a in TRPs members may contribute to the development of podocyte injury.

In conclusion, the present study suggested that miR-135a is a significant factor in podocyte injury. miR-135a expression was found to be upregulated in podocyte injury, while TRPC1 expression was downregulated. Ectopic expression of TRPC1 prevents podocyte injury by miR-135a. These findings provide novel insights into the role of miR-135a in glomerulopathies and support the development of a novel therapeutic strategy.

\section{Acknowledgements}

The authors wish to acknowledge the support of the Sichuan Province Department of Education Program, China (grant no. 16ZB0275, awarded to X.Y.). The present study was also supported by the fund research projects of Chengdu Medical College in Sichuan Province, China (grant no. CYTD15-03, awarded to Y.X.).

\section{References}

1. Nilius B and Szallasi A: Transient receptor potential channels as drug targets: From the science of basic research to the art of medicine. Pharmacol Rev 66: 676-814, 2014.

2. Barisoni L: Podocyte biology in segmental sclerosis and progressive glomerular injury. Adv Chronic Kidney Dis 19: 76-83, 2012.

3. Itoh M, Nakadate K, Horibata Y, Matsusaka T, Xu J, Hunziker W and Sugimoto $\mathrm{H}$ : The structural and functional organization of the podocyte filtration slits is regulated by Tjp1/ZO-1. PLoS One 9: e106621, 2014.

4. Shankland SJ: The podocyte's response to injury: Role in proteinuria and glomerulosclerosis. Kidney Int 69: 2131-2147, 2006.

5. Grishok A, Pasquinelli AE, Conte D, Li N, Parrish S, Ha I, Baillie DL, Fire A, Ruvkun G and Mello CC: Genes and mechanisms related to RNA interference regulate expression of the small temporal RNAs that control C. elegans developmental timing. Cell 106: 23-34, 2001.

6. Li X and He JC: An update: The role of Nephrin inside and outside the kidney. Sci China Life Sci 58: 649-657, 2015.

7. May CJ, Saleem M and Welsh GI: Podocyte dedifferentiation: A specialized process for a specialized cell. Front Endocrinol (Lausanne) 5: 148, 2014.

8. Gebeshuber CA, Kornauth C, Dong L, Sierig R, Seibler J, Reiss M, Tauber S, Bilban M, Wang S, Kain R, et al: Focal segmental glomerulosclerosis is induced by microRNA-193a and its downregulation of WT1. Nat Med 19: 481-487, 2013.

9. Lin CL, Lee PH, Hsu YC, Lei CC, Ko JY, Chuang PC, Huang YT, Wang SY, Wu SL, Chen YS, et al: MicroRNA-29a promotion of nephrin acetylation ameliorates hyperglycemia-induced podocyte dysfunction. J Am Soc Nephrol 25: 1698-1709, 2014.

10. Wu J, Zheng C, Fan Y, Zeng C, Chen Z, Qin W, Zhang C, Zhang W, Wang X,Zhu X, et al: Downregulation of microRNA-30 facilitates podocyte injury and is prevented by glucocorticoids. J Am Soc Nephrol 25: 92-104, 2014.

11. Shi S, Yu L, Zhang T, Qi H, Xavier S, Ju W and Bottinger E: Smad2-dependent downregulation of miR-30 is required for TGF- $\beta$-induced apoptosis in podocytes. PLoS One 8: e75572, 2013. 
12. Yang X, Wang X, Nie F, Liu T, Yu X, Wang H, Li Q, Peng R, MaoZ,Zhou Q, et al: miR-135 family members mediate podocyte injury through the activation of $\mathrm{Wnt} / \beta$-catenin signaling. Int J Mol Med 36: 669-677, 2015.

13. Zhang C, Chen X, Chen X, Wang X, Ji A, Jiang L, Sang F and Li F: miR-135a acts as a tumor suppressor in gastric cancer in part by targeting KIFC1. Onco Targets Ther 9: 3555-3563, 2016.

14. Wan X, Pu H, Huang W, Yang S, Zhang Y, Kong Z, Yang Z, Zhao P, Li A, Li T, et al: Androgen-induced miR-135a acts as a tumor suppressor through downregulating RBAK and MMP11, and mediates resistance to androgen deprivation therapy. Oncotarget 7: 51284-51300, 2016.

15. Yamada Y, Hidaka H, Seki N, Yoshino H, Yamasaki T, Itesako T, Nakagawa $\mathrm{M}$ and Enokida H: Tumor-suppressive microRNA-135a inhibits cancer cell proliferation by targeting the c-MYC oncogene in renal cell carcinoma. Cancer Sci 104: 304-312, 2013

16. Wu S, Lin Y, Xu D, Chen J, Shu M, Zhou Y, Zhu W, Su X, Zhou Y, Qiu P, et al: MiR-135a functions as a selective killer of malignant glioma. Oncogene 31: 3866-3874, 2012.

17. Nagel R, le Sage C, Diosdado B, van der Waal M, Oude Vrielink JA, Bolijn A, Meijer GA and Agami R: Regulation of the adenomatous polyposis coli gene by the miR-135 family in colorectal cancer. Cancer Res 68: 5795-5802, 2008.

18. Holleman A, Chung I, Olsen RR, Kwak B, Mizokami A, Saijo N, Parissenti A, Duan Z, Voest EE and Zetter BR: miR-135a contributes to paclitaxel resistance in tumor cells both in vitro and in vivo. Oncogene 30: 4386-4398, 2011.

19. Zhou W, Li X, Liu F, Xiao Z, He M, Shen S and Liu S: MiR-135a promotes growth and invasion of colorectal cancer via metastasis suppressor 1 in vitro. Acta Biochim Biophys Sin (Shanghai) 44: 838-846, 2012

20. Ni L, Saleem M and Mathieson PW: Podocyte culture: Tricks of the trade. Nephrology (Carlton) 17: 525-531, 2012.

21. Fogo AB: Animal models of FSGS: Lessons for pathogenesis and treatment. Semin Nephrol 23: 161-171, 2003.

22. Wang Y, Wang YP, Tay YC and Harris DC: Progressive adriamycin nephropathy in mice: Sequence of histologic and immunohistochemical events. Kidney Int 58: 1797-1804, 2000

23. Mundel P, Reiser J, Zúñiga Mejía Borja A, Pavenstädt $H$, Davidson GR, Kriz W and Zeller R: Rearrangements of the cytoskeleton and cell contacts induce process formation during differentiation of conditionally immortalized mouse podocyte cell lines. Exp Cell Res 236: 248-258, 1997.
24. Kolfschoten IG, van Leeuwen B, Berns K, Mullenders J, Beijersbergen RL, Bernards R, Voorhoeve PM and Agami R: A genetic screen identifies PITX1 as a suppressor of RAS activity and tumorigenicity. Cell 121: 849-858, 2005.

25. Bae GU, Lee JR, Kim BG, Han JW, Leem YE, Lee HJ, Ho SM, Hahn MJ and Kang JS: Cdo interacts with APPL1 and activates Akt in myoblast differentiation. Mol Biol Cell 21: 2399-2411, 2010.

26. Niehof M and Borlak J: HNF4 alpha and the Ca-channel TRPC1 are novel disease candidate genes in diabetic nephropathy. Diabetes 57: 1069-1077, 2008.

27. Chen K, Jin X, Li Q, Wang W, Wang Y and Zhang J: Association of TRPC1 gene polymorphisms with type 2 diabetes and diabetic nephropathy in Han Chinese population. Endocr Res 38: 59-68, 2013.

28. Zhang D, Freedman BI, Flekac M, Santos E, Hicks PJ, Bowden DW, Efendic S, Brismar K and Gu HF: Evaluation of genetic association and expression reduction of TRPC1 in the development of diabetic nephropathy. Am J Nephrol 29: 244-251, 2009.

29. Liu S, Guo W, Shi J, Li N, Yu X, Xue J, Fu X, Chu K, Lu C, Zhao J, et al: MicroRNA-135a contributes to the development of portal vein tumor thrombus by promoting metastasis in hepatocellular carcinoma. J Hepatol 56: 389-396, 2012.

30. Chen Y, Zhang J, Wang H, Zhao J, Xu C, Du Y, Luo X, Zheng F, Liu R, Zhang H, et al: miRNA-135a promotes breast cancer cell migration and invasion by targeting HOXA10. BMC Cancer 12: 111, 2012.

31. Wang Q, Wang Y, Minto AW, Wang J, Shi Q, Li X and Quigg RJ: MicroRNA-377 is up-regulated and can lead to increased fibronectin production in diabetic nephropathy. FASEB J 22: 4126-4135, 2008.

32. Navarro A, Diaz T, Martinez A, Gaya A, Pons A, Gel B, Codony C, Ferrer G, Martinez C, Montserrat E, et al: Regulation of JAK2 by miR-135a: Prognostic impact in classic Hodgkin lymphoma. Blood 114: 2945-2951, 2009.

33. Woudenberg-Vrenken TE, Bindels RJ and Hoenderop JG: The role of transient receptor potential channels in kidney disease. Nat Rev Nephrol 5: 441-449, 2009.

34. Reiser J, Polu KR, Möller CC, Kenlan P, Altintas MM, Wei C, Faul C, Herbert S, Villegas I, Avila-Casado C, et al: TRPC6 is a glomerular slit diaphragm-associated channel required for normal renal function. Nat Genet 37: 739-744, 2005. 